its only possible origin is off the plate. It is a question of imbalance between food intake and energy expenditure. Since food intake is regulated by appetite, Dr Morrison's paper is pertinent.

The treatment of obesity is largely a problem for dietetic practice, and it is fitting that Miss Rose should conclude this Symposium with an account of her unrivalled experience in this field. Obesity is a very prevalent disease; a proper nation-wide attack on it would employ far more dietitians than are at present registered with the British Dietetic Association.

\title{
Obesity and the control of food intake in experimental animals
}

\section{By S. D. Morrison, Institute of Physiology, University of Glasgow}

Obesity of any sort, in man or in animals, is immediately the result of a positive energy balance. A positive energy balance can occur from an excessive food (energy) intake, from a deficient energy output as heat or work, from a diminished ability to utilize absorbed energetic materials, or from any combination of these causes. This fact, being stated, does not advance the solution of the physiological problem which obesity poses. It does, however, underline the primary thermodynamic nature of the physical problem, and points the way to some of the possible experimental approaches to the study of obesity.

Over the past 20 years various techniques have been developed for the induction of obesity in experimental animals. Such obesity may be used to find what might be described as experimental analogues of human obesity syndromes. Ultimately a more rewarding approach would probably be to use these obesities not so much for the study of obesity as such, but as tools for the investigation of the mechanisms by which the normal individual maintains body-weight, and particularly the energetic part of body-weight, within very narrow limits without apparent effort or consciousness.

The many types of experimental obesity may be classified in a variety of ways: according to method of induction, according to the predominant origin of energy imbalance, or according to a variety of associated metabolic criteria such as glucose tolerance or insulin resistance. The main types of obesity are listed in Table $\mathbf{I}$ along with a qualitative estimate of the changes in energy intake and output which contribute to the obesity. Whatever their origin and detailed causation, all these obesities have in common that the source of the energy imbalance lies at least partly and often predominantly in an increased intake of food.

Mayer (1953) has proposed a dichotomy of experimental and of clinical obesities into 'regulatory' and 'metabolic' types. The basis of this separation is the proposition that some of the obesities, the 'regulatory' group, are the result of a primary breakdown in control of food intake, and others are the consequence primarily of an abnormality in the utilization of absorbed materials, the alteration in food intake being merely a reflection of the metabolic abnormality. This distinction is valuable, 
but it should not be allowed to obscure the fact that every type does show some increase in food intake.

\section{Table I. Experimental obesities induced in animals}

\begin{tabular}{|c|c|c|c|}
\hline & Method of Induction & $\begin{array}{l}\text { Change in } \\
\text { food intake }\end{array}$ & $\begin{array}{l}\text { Change in } \\
\text { energy expenditure }\end{array}$ \\
\hline \multirow{3}{*}{$\begin{array}{l}\text { Class } \\
\text { Regulatory }\end{array}$} & Surgical lesions of & & \\
\hline & hypothalamus & +++ & + \\
\hline & Goldthioglucose & $+t+$ & + \\
\hline \multirow[t]{5}{*}{ Mixed } & Avoidance conditioning & & \\
\hline & (forced feeding) & $++t$ & ? \\
\hline & Immobilization & + & --- \\
\hline & High-fat diet (genetic & & \\
\hline & interaction) & + & ? \\
\hline \multirow[t]{2}{*}{ Metabolic } & Genetic types (mice) & & \\
\hline & Obese hyperglycaemic & ++ & + \\
\hline \multirow[t]{4}{*}{ - } & Yellow obese & $+t$ & - \\
\hline & NZO strain obese & $+t$ & $?$ \\
\hline & ACTH-secreting tumour & + & - \\
\hline & Chronic insulin treatment & ++ & $?$ \\
\hline
\end{tabular}

The complex of co-ordinated motor activity which we call feeding is not continuous but starts and stops at intervals. In the normal animal the total intake of food over a long period is closely matched to that requirement which will supply the necessary energy expenditure and maintain the body-weight within relatively narrow limits. It appears obvious, then, that the feeding complex must be controlled by a sensory inflow through which some signal (or signals) starts the process of eating, which is stopped by the absence or change in strength of this signal or by other signals. In the regulatory obesities the error lies in the mechanism that responds to the signal. In the metabolic obesities the signal itself is altered or modified as a result of some other error. In either situation we want to know the mode of action of this entire feeding assembly, signal, sensory and motor mechanisms.

\section{Central control of feeding}

Erdheim (1904) suggested that the obesity of Fröhlich's syndrome arises from a neural lesion rather than from damage to the pituitary. Further work tended to confirm this view and to narrow the field of involvement to the hypothalamus. With Hetherington \& Ranson's (1939) demonstration that obesity could be produced in rats by lesions in the hypothalamus without any involvement of the pituitary, the thesis was established. This form of obesity has now been produced in mice, rats, cats and monkeys.

The precise localization of the effective lesions is in the ventro-medial nucleus of the hypothalamus (Fig. I), and the obesity results from a great increase of food intake unbalanced by a corresponding increase in energy expenditure (Brobeck, Tepperman \& Long, 1943). The body-weight can increase to about twice that of a control, and the weight gain is almost entirely accounted for by fat deposition. There is no change in the total body nitrogen and little change in water. The hyperphagia, which appears immediately after the lesion, persists but with falling intensity until the animal's weight reaches a new relatively constant level. At this stage the food intake 
falls to a level only slightly above normal. If the animal is now starved the bodyweight returns to normal. But this induces the reappearance of hyperphagia, and, if food is again available, there is a rapid rise in body-weight to a stable obese level. The obese animal fasted to normal body-weight has a normal body composition.
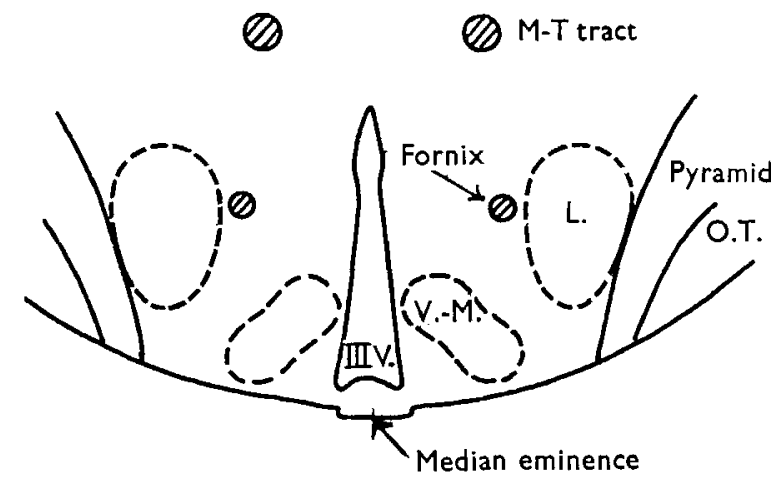

Fig. I. Coronal section through hypothalamus of the rat in plane of the median eminence, showing positions of ventro-medial (V.-M.) and lateral (L.) ateas. M-T tract, mamillo-thalamic tract; III V., third ventricle; O.T., optic tract.

Anand \& Brobeck (195I) discovered another area in the lateral hypothalamus (Fig. I) influencing feeding, bilateral destruction of which produced complete aphagia. They proposed that the hypothalamic control of feeding is the resultant of two centres acting reciprocally: a 'satiety' centre, destruction of which causes hyperphagia and which in normal operation limits the feeding activity, and a 'feeding' centre, destruction of which causes aphagia and in normal operation actuates or maintains feeding activity or does both.

Any stable control system must have (a) a sensory input, (b) a central integrating organization, (c) an effector or response mechanism and (d) a feed-back device, i.e. some part of the input or sensory side of the system must be capable of modification by some characteristic of the response or output side. The discovery of the influence of the two hypothalamic centres has established that the hypothalamus is one of the central integrative mechanisms, and probably the most important. (Other areas have been found which can drastically modify food intake.) The existence of these centres throws no immediate light, however, upon the other three necessary components of the control system and does not allow any conclusion to be reached even about the way in which the centres exert their control.

\section{The sensory inflow}

What makes an animal start eating? What makes it stop eating? The same signal may operate for each but at a different level, i.e. above and below a certain threshold, or there may be different signals for the two events. It is obvious that the senses of sight, taste and smell modify feeding behaviour, and in the hyperphagic and even aphagic animals these effects may be accentuated, but there is abundant evidence that they only modify a more primitive control system and are not the prime movers. 
There is, at present, no conclusive, or even very convincing, evidence concerning the nature of the signals either for starting or for stopping eating. As a result, a number of hypotheses has been advanced, each of which is to some degree consistent with the evidence and each contributing to extension and enlargement of the evidence. The detailed evidence for and criticism of these hypotheses have been presented many times (Mayer, 1955) and only the bare essentials of each will be discussed here.

Gastric contractions. The earliest hypothesis supported experimentally was that the rhythmic contractions of the empty stomach, known as hunger contractions, brought hunger to the conscious level through ingoing fibres in the vagus nerve and so initiated eating. This theory was first presented in organized form by Cannon \& Washburn (I $1 \mathrm{I} 2)$. Their proposition was confined, however, to claiming that hunger contractions were the origin of the subjective sensation of hunger which, in turn, would initiate eating. They explicitly did not represent hunger contractions as the explanation of all food intake. The theory has been almost totally rejected in recent years, as subjective hunger, appetite and a controlled food intake still exist in the gastrectomized animal and in the animal with all extrinsic nerves to the stomach divided. Certainly it is right that this theory should be rejected as an explanation of total food intake. But, that a grossly abnormal animal can control its food intake satisfactorily without this mechanism does not mean that in the normal animal it plays no part. The evidence for the association of hunger contractions with food drive is massive and has recently been further supported by the finding that feeding activity can be induced by electrical stimulation of the dorsal vagal nucleus in the medulla (Larsson, 1954).

Gastric distention. This hypothesis proposes that the distension of the stomach resulting from food or other material placed in it signals the termination of eating. Countless experiments by a variety of different techniques have demonstrated that an animal experiences some measure of satiety from the presence of material in the stomach. Two features of this work are particularly worth noting. First, no matter how great an amount of food is placed directly into the stomach, there always seems to remain some unsatiated fraction that arouses eating when food is presented for oral consumption. This unsatiated residue, however, decreases with the time that the procedure is maintained, and is finally much smaller than would appear in a shortterm experiment (Janowitz \& Hollander, I955). Secondly, over short periods, the reward or satiation value of material in the stomach is not entirely dependent on its having nutritive value. The distension of a balloon in the stomach of a rat produces almost the same degree of satiation, as measured by the work it will do to obtain food by mouth, as the presence in the stomach of an equal volume of milk introduced by fistula (Miller, 1955).

It is evident that peripheral mechanisms involving certainly gastric distension and probably hunger contractions play a part in the normal control of food intake. The control, however, is incomplete, to some extent indiscriminate, and is dispensable without notable disadvantage. Experiments with diets diluted with inert material (Adolph, 1947) show that an animal is in fact able to discriminate nutrient from 
non-nutrient materials and with progressive dilution of the diet will, up to a limit, increase its bulk intake to maintain its nutrient intake constant. These limitations imply the existence of some signal which is a function of the nutrient content of the diet and not of its bulk.

Thermostatic hypothesis. This theory, largely developed by Brobeck (1948, 1957), is based on the following propositions and experimental findings: ( $I$ ) one of the common factors in the metabolic response to eating is heat production, (2) after or during eating there is peripheral vasodilatation indicating a temperature-control response, and ( 3 ) the hypothalamus is known to be involved in temperature regulation and there is evidence that thermo-receptors exist in the hypothalamus. It is proposed, therefore, that a type of thermal receptor similar to that which may operate in temperature regulation is also effective in regulation of feeding. To take account of the fact that animals do not regulate their intake on different diets very closely or accurately for energy intake, it is further proposed that the additional heat production during and after eating, that is the specific dynamic action (s.D.A.), is the effective signal. Fall in heat production initiates eating, rise in heat production beyond a certain threshold terminates it.

One objection to this theory lies in the technical difficulty of testing it. Many of the techniques that can conveniently be applied to investigation of the central control of temperature regulation are not easily adaptable to studies of food intake which require conscious animals over relatively long periods. Also the use of S.D.A. as a major pillar of the hypothesis is a weakness. S.D.A. is an elusive function experimentally and many situations can be proposed where S.D.A. and satiety do not go hand in hand.

Glucostatic hypothesis. This theory, largely developed by Mayer (1953), is also based on the concept of a common metabolic factor, in this instance blood glucose. The relationship of hypoglycaemia to subjective hunger has been known for many years. The utilization of glucose is postulated as the effective determinant and this does not necessarily follow the absolute glucose level (cf. diabetes mellitus). The arterio-venous (a.-v.) glucose difference is proposed as the effective signal. A high a. -v. difference indicates a high peripheral utilization of glucose and, ipso facto, a high availability of glucose. A low a.-v. difference indicates a low utilization of glucose. The theory, up to this point, can be shown to be not inconsistent with most of the evidence. Direct attempts to test the relationship between a.-v. glucose differences and food drive have yielded equivocal results but they may be in part due to the use, for technical reasons, of capillary-venous differences as a substitute. The theory, however, requires a 'glucoreceptor' sensitive to an a.-v. glucose difference. Being proposed, this 'glucoreceptor' has been sited in the hypothalamus.

At this point it is necessary to impose the first of two limitations on the interpretation of this and other 'central' theories of control. It is beyond doubt that the major central control of food intake lies in the hypothalamus. It is not beyond doubt that the regulating centres are themselves the receptors. There is some inconclusive evidence on this point, but it is neither an experimental nor a logical necessity and may, at the present stage, be too cramping a restriction.

18 (2) 5 
The major single objection to the glucostatic theory (within the limits which its authors have imposed on it, that is as a short-term control) is the fact that, whereas ingestion of glucose raises the blood sugar and also reduces the complex of sensation and food drive associated with hunger, as also does intravenous glucagon (a hyperglycaemic agent), the intravenous administration of glucose has not been demonstrated to relieve any of the immediate manifestations of hunger (cf. Morrison, Lin, Eckel, Van Itallie \& Mayer, 1958).

The further criticism that the 'glucoreceptors' have not been demonstrated as 'things' could with equal force be levelled at the proposed receptors in any of the other hypotheses, including the peripheral gastric theories.

All the mechanisms so far mentioned have in common that the proposed effective signals are highly labile. Errors of estimate from day to day or, in all the systems described, from meal to meal, might cancel out but might be cumulative. In an analysis of the normal pattern of control of food intake in rabbits, Gasnier \& Mayer (I939) found that the further the body substance drifted from the ideal weight the more likely was the next alteration in food intake to be restorative towards the ideal weight and, if restorative, also to be large. This finding suggests that there must be a mechanism able to correct accumulating errors made by the more labile shortterm mechanisms.

Lipostatic hypothesis. The animal with lesions in the ventro-medial hypothalamus is hyperphagic and so becomes obese, but it is not infinitely hyperphagic and does not become infinitely obese. Its body-weight reaches a plateau after some time at a new high level. On the basis of this observation, Kennedy (r953) proposed that the accumulation of lipid reserves acts in some way as a control mechanism.

A recent report by Hervey ( 1959 ) indicates that the normal as well as the hypothalamic obese animal is sensitive to this mechanism. When lesions were made in the hypothalamus of one member of a parabiotic pair of rats the partner with lesions became hyperphagic and obese. The non-operated partner became progressively more emaciated.

\section{Integration and speculation}

Whereas the effect of lesions in the ventro-medial hypothalamus shows an upper limit, lesions in the lateral hypothalamus produce complete and persisting aphagia with death of the animal from starvation. This aphagia is invariably associated with complete adipsia in the rat but it is nevertheless a completely independent effect (Morrison \& Mayer, 1957). Spontaneous recovery of food intake can sometimes occur in these animals but appears to be the result of incomplete destruction of the lateral area (Mayer \& Morrison, 1958). It appears, then, that none of the various suggested mechanisms for control of food intake is able to act through the ventromedial ('satiety') centre alone, whereas the lipostatic mechanism at least could act through the lateral area alone.

The increased food intake in hyperphagic animals appears to be achieved largely through an increase in the size of the meal taken, but the meals are still clearly 
separable (Teitelbaum \& Campbell, 1958). Since the meal can still be started and stopped, it is probable that all of the short-term control mechanisms can also operate through the lateral ('feeding') centre alone but at a higher threshold than on the 'satiety' centre. As the hunger contractions have never been proposed to be anything other than an initiating signal it seems unnecessary to propose that they operate on more than the 'feeding' centre. Although quite ineffective, they appear to occur normally in the aphagic animal (Morrison, unpublished work).

From these relations, then, it is possible to propose that the ventro-medial hypothalamic centre functions as a receiving station for the sensory inflow at a low threshold, i.e. a high sensitivity. Its outflow could be exclusively an inhibition on the lateral area. The lateral area has (I) an inhibitory inflow from the ventro-medial area, (2) a sensory inflow at a high threshold (low sensitivity) and in the opposite sense from the ventro-medial centre (i.e. inhibition of the ventro-medial centre implies excitation of the lateral centre and vice versa), and (3) exclusive control over the motor outflow. This general scheme is summarized in Fig. 2.

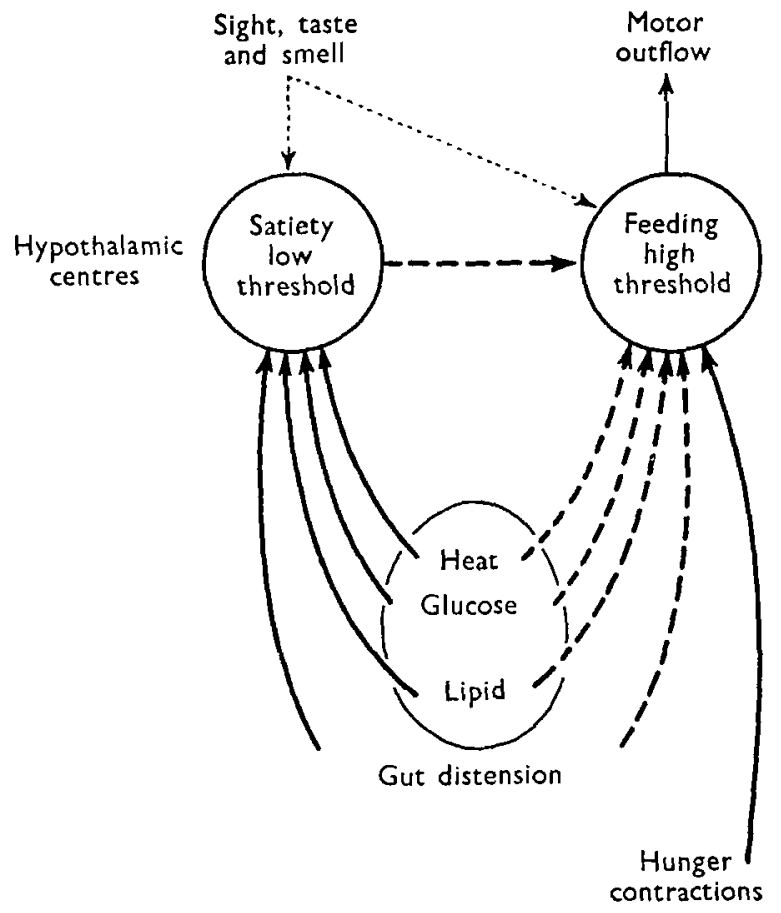

Fig. 2. Schematic summary of the possible modes of action of the various mechanisms postulated in the control of food intake. - - excitation; - - - , inhibition.

Here it is necessary to impose the second general limitation on the interpretation of these hypotheses. There is, at present, no proof that the actual signals to which the sensory elements respond are in fact heat, glucose or lipid. These are simply possible variates which may be used to measure the signal. The question may fairly 
be asked, is any of these variates an actual signal, or may each not be a reflection of other real signals or even of a common signal?

Any single control hypothesis would have to propose a feature common to each of the proximate principles, and would also have to satisfy the requirements of shortterm and long-term control. It seems likely that the conflict between short-term and long-term control could be resolved by the differences in threshold of the two centres, without distinct mechanisms being invoked. A common signal, however, is more easily proposed than found. At no point are the metabolic paths of protein, fat and carbohydrate coincident although they do overlap to some extent. At this stage it is convenient to accept Brobeck's (1957) suggestion of a 'multiple factor' control, by analogy with the control of respiration, as a working basis for further investigation. It would be helpful, however, if the number of mechanisms involved and receptors to be found could be reduced. Multiple-factor theories, which are entirely empirical, are always unsatisfactory.

\section{REFERENCES}

Adolph, E. F. (1947). Amer. F. Physiol. r5r, i 10.

Anand, B. K. \& Brobeck, J. R. (1951). Yale ¥. Biol. Med. 24, I23.

Brobeck, J. R. (1948). Yale F. Biol. Med. 20, 545.

Brobeck, J. R. (1957). Yale F. Biol. Med. 29, 565 .

Brobeck, J. R., Tepperman, J. \& Long, C. N. H. (1943). Yale F. Biol. Med. 15, 33 I.

Cannon, W. B. \& Washburn, A. L. (Igra). Amer. F. Physiol. 29, 441.

Erdheim, J. (1904). S.B. Akad. Wiss. Wien, Abt. iii, I13, 537.

Gasnier, A. \& Mayer, A. (1939). Ann. Physiol. Physicochim. biol. 15, 57.

Hervey, G. R. (1959). F. Physiol. 145, 336.

Hetherington, A. W. \& Ranson, S. W. (1939). Proc. Soc. exp. Biol., N.Y., 41, 465.

Janowitz, H. D. \& Hollander, F. (1955). Ann. N.Y. Acad. Sci. 63, 56.

Kennedy, G. C. (1953). Proc, roy. Soc, B, 140, 578.

Larsson, S. (1954). Acta physiol. scand. 32, suppl. 115.

Mayer, J. (1953). Physiol. Rev. 33, 472.

Mayer, J. (1955). Nutr. Abstr. Rev. 25, 597.

Mayer, J. \& Morrison, S. D. (1958). F. Physiol. r43, 4I $P$.

Miller, N. E. (1955). Ann. N.Y. Acad. Sci. 63, I4I.

Morrison, S. D., Lin, H. J., Eckel, H. E., Van Itallie, T. B. \& Mayer, J. (1958). Amer. F. Physiol. 193, 4. Morrison, S. D. \& Mayer, J. (1957). Amer. F. Physiol. I91, 248.

Teitelbaum, P. \& Campbell, B. A. (1958). F. comp. physiol. Psychol. 51, 135.

\section{The measurement of body fat in man}

By J. M. Tanner, Institute of Child Health, University of London, Hospital for Sick Children, Great Ormond Street, London, W.C.I

\section{Introduction}

The methods available for estimating body fat in man are ( $\mathrm{r}$ ) anthropometric, by measurement of the thickness of subcutaneous tissue by calipers or by radiography; (2) direct physical and chemical (not applicable to living persons), by fat-solvent extraction of the whole body or parts of it; (3) indirect physical and chemical, by measurement of body density or body water, or a combination of the two, various assumptions being made; (4) indirect anthropometric. 